\title{
The Impact of Community Structure on the Convergence Time of Opinion Dynamics
}

\author{
An Lu, Chunhua Sun, and Yezheng Liu \\ School of Management of Hefei University of Technology, Hefei, Anhui Province 230009, China \\ Correspondence should be addressed to Yezheng Liu; liuyezheng@hfut.edu.cn
}

Received 14 December 2016; Revised 28 February 2017; Accepted 19 April 2017; Published 1 June 2017

Academic Editor: Lu-Xing Yang

Copyright (C) 2017 An Lu et al. This is an open access article distributed under the Creative Commons Attribution License, which permits unrestricted use, distribution, and reproduction in any medium, provided the original work is properly cited.

\begin{abstract}
We analyze the convergence time of opinion dynamics in a social network with community structure. Using matrix analysis, we prove that the convergence time is determined by the second largest eigenvalue modulus. This modulus is close to 1 if the social influence matrix is nearly uncoupled. Furthermore, we discuss and analyze the factors of community structure affecting the convergence time.
\end{abstract}

\section{Introduction}

Community structure is ubiquitous in reality. It means that many social networks can be divided into some groups such that the connection within each group is dense, while connection between groups is very sparse. Community structure is always relevant to many social and biological phenomena. Previous works have shown that community structure affects the evolution of macroscopic phenomena taking place on a network, such as synchronization $[1,2]$, the spread of epidemics [3,4], rumors [5], and opinion dynamics [6-8].

In opinion dynamics, various versions of the opinion models have been proposed. First types are discrete opinion models, among which are the Sznajd model [9], the voter model $[10,11]$, the majority rule model [12], and the social impact model [13]. Other models are continuous, using concepts and methods based on ideas from statistical physics or control theory. The most famous are bounded confidence model [14, 15] and an earlier model, Degroot model [16], including some agent-based models such as [17].

There are two interesting questions in opinion dynamics. First, under what conditions will the opinions' updating processes converge to a well-defined limit? The second question is about the convergence time or convergence rate problem; that is, how quickly the consensus is reached if opinions can converge ultimately. Previous works have shown that the convergence time is determined by the topology of social network and the updating rule of individuals' opinion. For example, in bounded confidence model [15], the convergence time is determined by bounded confidence parameter, which is expressed by a real number $\varepsilon$, such that an agent, with opinion $x$, only interacts with its peers whose opinion lies in the range of $[x-\varepsilon, x+\varepsilon]$. In another bounded confidence model, Deffuant model [14], the convergence time is only determined by convergence parameter $\mu$. In [18], the authors studied the effects of adding shortcuts connecting randomly chosen pairs of sites in a regular lattice on the consensus time, using a local majority updating rule. They showed that the consensus time dropped sensitively with the addition of a small number of shortcuts. In [19], the author introduced a two-state opinion dynamics model where agents evolve by majority rule, finding that consensus is reached in a time that scales to $\log N$, where $N$ is the number of agents. On finite-dimensional lattices, where a group is a contiguous cluster, the consensus time fluctuates strongly between realizations and grows as a dimension-dependent power of $N$. The upper critical dimension appears to be larger than 4. In [20] the authors found that, for the voter model, if the network is with an arbitrary but uncorrelated degree distribution, the convergence time $T_{N}$ scales as $N \mu_{1}^{2} / \mu_{2}$, where $\mu_{k}$ is the $k$ th moment of the degree distribution and $N$ is the size of the network. In [21], the authors found in 
a coevolving network that if the number of committed agents added exceeds a critical value, the consensus time growth becomes logarithmic in network size $N$. Slight changes in the interaction rule can produce strikingly different results of consensus time.

Although it is shown that interaction rules or network topologies have an important impact on the evolution of group opinions, the continuous opinion dynamics issue with community structure is still not well understood. Unlike all the research above, here we mainly discuss the impact of a community structure on convergence time, which applies to situations of how we control the opinion evolution in a social network with community structure. First, through matrix analysis method, we prove that the convergence time is determined by the second largest eigenvalue modulus of social influence matrix. Furthermore, we prove that this modulus is close to 1 if the social influence matrix is nearly uncoupled. Second, in our model the influence of interpersonal is random, which is closer to reality. We propose the concept, distribution of interpersonal influence, which can better describe the interaction situations. We examine this issue from three points of view: the number of nodes connected (including the number of nodes connected between subgroups, the number of nodes connected within subgroups, and the connection density between subgroups), the size of subgroups, and influence distribution. We find that the number of connections between subgroups and the number of subgroups within the subgroups exert a strong influence on the convergence time. In addition, the impact of distribution of influence on the convergence time shows the following fact: the convergence time of the group opinions in an autocratic society is longer than that in a democratic society in average, but various connection patterns may bring much more uncertainty.

The remaining of this paper is organized as follows: in Section 2, we discuss the proposed model in detail. Then in Section 3 we analyze the impact of community structure on the consensus time. Section 4 concludes this paper.

\section{Continuous Opinion Dynamics Model}

2.1. Notations, Assumptions, and the Opinion Dynamic Model. This section introduces the notations and the assumptions and defines the consensus time of continuous opinion dynamics.

Mathematically, a social network with community structure can be characterized by a big graph in which the nodes represent people, and the edges evaluate their relation strength.

We consider a set of $n$ individuals in a social network. $V=\{1,2, \ldots, n\}$. A relation $E \subseteq V \times V$ models the interactions between individuals. We assume the relationship is mutual $((i, j) \in E$ if and only if $(j, i) \in E) . V$ is the set of vertices and $E$ is a set of edges of undirected graph $G=(V, E)$, describing the social network of individuals. Each individual has an opinion modeled by a real number $x_{i}(t) \in R$. Initially, individual $i$ has an opinion $x_{i}(0)$ independent from others. Then, at every time step, the individuals update their opinion by taking a weighted average of their own opinion and opinions of others

$$
x_{i}(t+1)=\sum_{j=1}^{n} a_{i j}(t) x_{j}(t)
$$

with the coefficients $a_{i j}(t)$ satisfying

$$
\begin{aligned}
& \quad \forall i, j \in V, a_{i j}(t) \neq 0 \Longleftrightarrow j \in\{i\} \cup\left\{N_{i}(t)\right\}, \\
& \sum_{j=1}^{n} a_{i j}(t)=1,
\end{aligned}
$$

where the coefficient $a_{i i}$ denotes the force of self-confidence of individual $i$ and $a_{i j}$ denotes to what extent individual $i$ is affected by individual $j . N_{i}(t)$ denotes the neighborhood of individual $i$ at time $t$ step.

Let $X(t)=\left(x_{1}(t), x_{2}(t), \ldots, x_{n}(t)\right)^{T}, A(t)=\left(a_{i j}(t)\right)_{n \times n}$; thus, the group opinion dynamics can be written as follows:

$$
X(t+1)=A(t) X(t),
$$

where $A$ is a row stochastic and nonnegative matrix. In this following, we call it social influence matrix whose entry $a_{i j}$ represents the influence strength from individuals $j$ to $i$.

In this paper, we assume that (1) $G$ is static and strongly connected; (2) the relationship and interaction between individuals are mutual; (3) every individual has a little selfconfidence. Under these conditions, it is easy to find that $A$ is constant and row stochastic matrix. The diagonal entries in $A$ are all positive, and zero-entries in $A$ are symmetric. Using nonnegative matrix theories, we can get some properties as follows.

\section{Proposition 1. Consensus will be reached ultimately.}

Proof. Under assumption (1), it can easily be seen that $A$ is an irreducible, row stochastic and nonnegative matrix. The summation of each row is equal to 1 . Thus, the spectral radius of $A$ denoted by $\rho(A)=1 . \alpha_{1}=(1,1, \ldots, 1)^{T}$ is an eigenvector of $\lambda=1$. Since all the diagonal entries are positive, using Perron-Frobenius theory, we can get $A$ is primitive. The algebraic multiplicity of $\lambda=1$ is equal to 1 . So the spectrum of $A$ can be denoted by

$$
\operatorname{spec}(A)=\left\{\lambda_{i}|| \lambda_{n}|\leq| \lambda_{n-1}|\leq \cdots \leq| \lambda_{2} \mid<\lambda_{1}=1\right\} .
$$

For any initial opinion vector $X(0)=\left(x_{1}(0), x_{2}(0)\right.$, $\left.\ldots, x_{n}(0)\right)^{T}$, it can be written as a linear combination as follows:

$$
X(0)=\sum_{i=1}^{n} k_{i} \alpha_{i}
$$

where $\alpha_{i}$ is eigenvector corresponding to the eigenvalue $\lambda_{i}$ of $A$. Thus, for all $t>0, \alpha_{i}^{t}$ is an eigenvector corresponding to the eigenvalue $\lambda_{i}^{t}$ of $A^{t}$. Since $\left|\lambda_{n}\right| \leq\left|\lambda_{n-1}\right| \leq \cdots \leq\left|\lambda_{2}\right|<\lambda_{1}=1$, it follows that

$$
\lim _{t \rightarrow \infty} X(t)=\lim _{t \rightarrow \infty} A^{t} \cdot X(0)=\lim _{t \rightarrow \infty} A^{t} \sum_{i=1}^{n} k_{i} \alpha_{i}
$$




$$
\begin{aligned}
& =\lim _{t \rightarrow \infty}\left(\sum_{i=1}^{n} k_{i} \alpha_{i} \cdot \lambda_{i}^{t}\right)=\lim _{t \rightarrow \infty}\left(k_{1} \alpha_{1}+o(1)\right) \\
& =\left(k_{1}, k_{1}, \ldots, k_{1}\right)^{T} .
\end{aligned}
$$

So the opinions converge to a constant vector, whose components are all equal. That means the consensus can be reached, and the convergence time is consensus time.

Remark. Assumption (2) is not necessary condition but sufficient for convergence of opinions. For example, if $A=$ $\left(\begin{array}{ccc}0 & 0.5 & 0.5 \\ 1 & 0 & 0 \\ 0 & 1 & 0\end{array}\right)$, we can get $\lim _{t \rightarrow \infty} A^{t}=\left(\begin{array}{ccc}0.4 & 0.4 & 0.2 \\ 0.4 & 0.4 & 0.2 \\ 0.4 & 0.4 & 0.2\end{array}\right)$, so opinions converge to a fixed vector, but the diagonal of $A$ is not positive.

Proposition 2. The convergence time is determined by the second largest eigenvalue modulus $\left|\lambda_{2}\right|$.

Proof. First we introduce the definition of convergence time:

$$
t^{*}=\inf \left\{t_{0} \mid \forall t>t_{0},\|X(t)-X\| \leq \delta\right\},
$$

where $\delta$ can be arbitrarily small, $X(t)$ represents a series of vector, and $X$ is the limit of $X(t)$.

$\|\cdot\|$ represents a vector norm (1-norm, 2-norm, or $\infty$ norm). If $\|X(t)-X\|<\delta$, it follows that

$$
\left\|k_{2} \alpha_{2} \cdot \lambda_{2}^{t}+o\left(\lambda_{2}^{t}\right)\right\| \leq \delta
$$

when $\delta$ is small enough, $o\left(\lambda_{2}^{t}\right)$ can be omitted. So (7) can be rewritten as $\left\|k_{2} \alpha_{2} \cdot \lambda_{2}^{t}\right\| \leq \delta$.

Thus, we can get

$$
t \geq \frac{-c \ln \delta}{\ln \left(1 /\left|\lambda_{2}\right|\right)}=\frac{-c \ln \delta}{-\ln \left|\lambda_{2}\right|}
$$

satisfying (7). So the convergence time $t^{*}=-c \ln \delta /-\ln \left|\lambda_{2}\right|$, where $c$ is a constant determined by the initial opinion vector and eigenvector of weighted matrix $A$.

From the analysis above, it is obvious that the larger $\left|\lambda_{2}\right|$ is, the longer the consensus time is and vice versa. For simplicity, we analyze the convergence time only by the second largest eigenvalue modulus $\left|\lambda_{2}\right|$ or $-\ln \left|\lambda_{2}\right|$.

Proposition 3. For a nonnegative row stochastic partitioned matrix $A=\left(\begin{array}{ll}B & D \\ E & C\end{array}\right)_{n \times n}$, where $B=\left(b_{i j}\right)_{n_{1} \times n_{1}}, C=\left(c_{i j}\right)_{n_{2} \times n_{2}}, n=$ $n_{1}+n_{2}$. Let $\sigma(A)$ be the summation of entries in $D$ and $E$; then for any $\varepsilon>0, \exists \delta>0$, if $|\sigma(A)|<\delta$, satisfying ||$\lambda_{2}|-1|<\varepsilon$.

Proof. First, we prove that if all the entries in a matrix change a little, the eigenvalues of the new matrix will also change. Denote $B$ by

$$
\left(\begin{array}{cccc}
b_{11} & b_{12} & \cdots & b_{1, n_{1}} \\
b_{21} & b_{22} & \cdots & b_{2, n_{1}} \\
\vdots & \vdots & \vdots & \vdots \\
b_{n_{1}, 1} & b_{n_{1}, 2} & \cdots & b_{n_{1}, n_{1}}
\end{array}\right)
$$

step $1 B$ is transformed into

$$
B^{(1)}=\left(\begin{array}{cccc}
b_{11}+\Delta^{(1)} & b_{12} & \cdots & b_{1, n_{1}} \\
b_{21} & b_{22} & \cdots & b_{2, n_{1}} \\
\vdots & \vdots & \vdots & \vdots \\
b_{n_{1}, 1} & b_{n_{1}, 2} & \cdots & b_{n 1, n_{1}}
\end{array}\right) .
$$

Thanks to the continuity of eigenvalues, we can get that, for any $\varepsilon>0$, there exists $\delta^{(1)}>0$, if $\left|\Delta^{(1)}\right|<\delta^{(1)}$, satisfying $\left|\lambda\left(B^{(1)}\right)-\lambda(B)\right|<\varepsilon$. For step 2 when $B^{(1)}$ is transformed into

$$
B^{(2)}=\left(\begin{array}{cccc}
b_{11}+\Delta^{(1)} & b_{12}+\Delta^{(2)} & \cdots & b_{1, n 1} \\
b_{21} & b_{22} & \cdots & b_{2, n 1} \\
\vdots & \vdots & \vdots & \vdots \\
b_{n 1,1} & b_{n 1,2} & \cdots & b_{n 1, n 1}
\end{array}\right) \text {, }
$$

we can get that, $\forall \varepsilon>0, \exists \delta^{(2)}>0$, if $\left|\Delta^{(2)}\right|<\delta^{(2)}$, $\left|\lambda\left(B^{(2)}\right)-\lambda\left(B^{(1)}\right)\right|<\varepsilon$. Repeat this process, at the last step, $\exists \delta^{\left(n_{1}^{2}\right)}>0$, if $\left|\Delta^{\left(n_{1}^{2}\right)}\right|<\delta^{\left(n_{1}^{2}\right)},\left|\lambda\left(B^{\left(n_{1}^{2}\right)}\right)-\lambda\left(B^{\left(n_{1}^{2}-1\right)}\right)\right|<\varepsilon$, so for any $\varepsilon>0$, there exists $\delta^{\prime}=\min \left(\delta^{(1)}, \delta^{(2)}, \ldots, \delta^{\left(n_{1}^{2}\right)}\right)$, if $\left|\Delta^{(1)}\right|,\left|\Delta^{(2)}\right|, \ldots,\left|\Delta^{\left(n_{1}^{2}\right)}\right|<\delta^{\prime}$, satisfying $\left|\lambda\left(B^{\left(n_{1}^{2}\right)}\right)-\lambda(B)\right|<n_{1}^{2} \varepsilon$.

That is to say, if the matrix perturbation is small enough, the eigenvalues will vary little accordingly. Secondly, let $A^{\prime}=$ $\left(\begin{array}{cc}B^{\prime} & 0 \\ 0 & C^{\prime}\end{array}\right)_{n \times n}$. So it is reducible, and naturally the spectral of $A^{\prime} \operatorname{spec}\left(A^{\prime}\right)=\operatorname{spec}\left(B^{\prime}\right) \cup \operatorname{spec}\left(C^{\prime}\right)$. If $\sigma(A)$ is small enough, then all the entries in $D$ and $E$ are also small. When $\sigma(A)$ trends toward 0 , the entries in $B$ and $C$ must change a little accordingly to keep $A$ as a row stochastic matrix. Without loss of generality, we can assume only one element $d_{11}>0$ in matrix $D$ (or $c_{11}$ in $C$ ). For these reasons, we can obtain that, for any $\varepsilon>0$, there exists $\delta$, if $d_{11}<\delta,\left|\lambda(A)-\lambda\left(A^{\prime}\right)\right|<\varepsilon$, since $d_{11}<\sigma(A)$; if $\sigma(A)<\delta$, then, $d_{11}<\delta$, $\left|\lambda(A)-\lambda\left(A^{\prime}\right)\right|<\varepsilon$ or specially ||$\lambda_{2}|-1|<\varepsilon$. That is to say, if $\sigma(A) \rightarrow 0$, then the second largest eigenvalue modulus is nearly 1 . The inverse of Proposition 3 has been proved by [22]. If second largest eigenvalue is sufficiently close to 1 , then $A$ is nearly uncoupled.

A social network with community structure means partitioned matrix $A$ with $\sigma(A)$ is very small. According to the above analysis, if individuals in different subgroups of a society rarely interact with each other, then $\lambda_{2}$ will be very close to 1 . As a result, it is hard to reach a consensus.

\section{The Impact of Community Structure on Convergence Time}

Although we have some analytical results of the second largest eigenvalue modulus of the social influence matrix with community structure, the exact results depend on entries of this matrix. Different network structures or different influence distributions will affect the second largest eigenvalue modulus, which in turn affects the convergence time of the group opinion. Therefore, the analysis of the impact of community structure on the convergence of time includes the following three aspects: the number of connection nodes, the size of subgroups, and the distribution of interpersonal influence. 


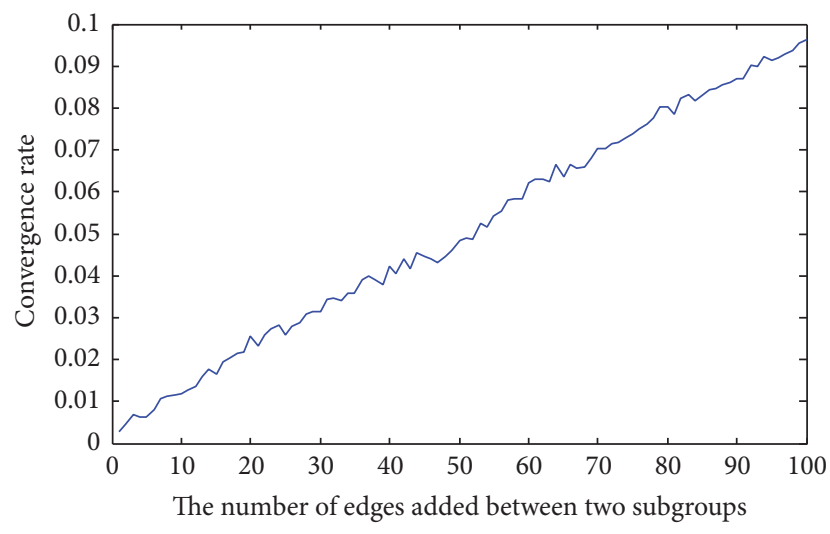

Figure 1: The relationship between the number of edges added between two subgroups and the convergence time.

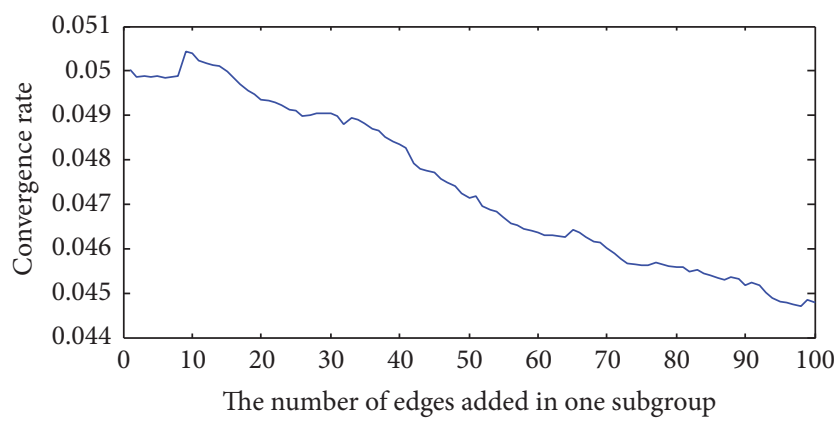

FIGURE 2: The relationship between the number of edges added in one subgroup and the convergence time.

3.1. The Number of Connection Nodes. First of all, using the famous WS model [23], we build a social network containing two subgroups, respectively. Each social network is a small world. To ensure the whole network is linked, the nodes between subgroups are connected sparsely and randomly. And then we investigate whether the increasing of connection can influence the convergence time.

Figure 1 shows that the convergence rate of opinion evolution is highly dependent on the connection pattern between subgroups. If the number of connections or connection densities between subgroups increases, the convergence rate of the group opinions is faster. At the beginning, when there is only one connection between subgroups, there is almost no connection between two subgroups, and the convergence rate is almost zero. This is the result of the aforementioned Proposition 3, and in this case, the social influence matrix associated the social network is nearly uncoupled. So consensus is hardly reached. However, when the number of connections (or densities) between the groups increases, for example, when the number of nodes increases to 10 pairs, the second largest eigenvalue modulus $\left|\lambda_{2}\right|$ will reduce from 0.9972 to 0.9881 . When the number of connections nodes goes up to $100,\left|\lambda_{2}\right|$ will drop to 0.9081 accordingly.

However, the convergence rate in Figure 2 declines with the rise of the number of internal connections in one subgroup. But the rate of weakening is much slower than

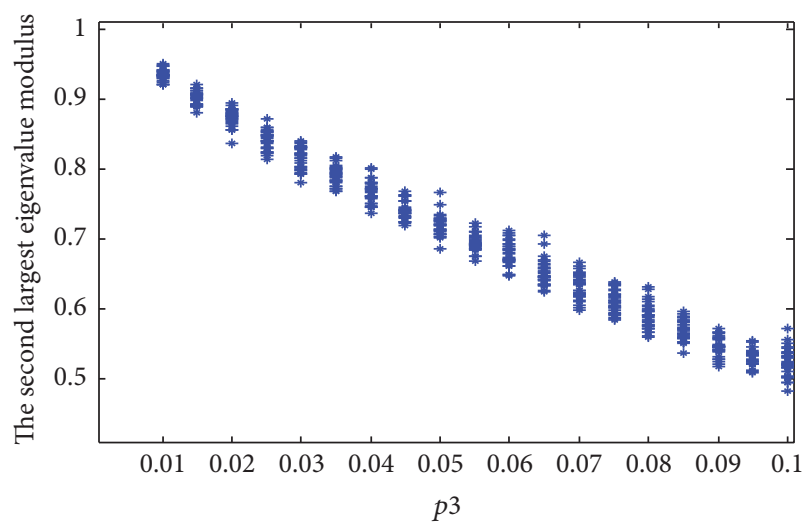

FIGURE 3: Example of the frequency of interaction between different subgroups impact on the second largest eigenvalue modulus, with $n_{1}=100, n_{2}=50, p_{1}=0.2$, and $p_{2}=0.4$ (repeatedly calculating 30 times).

that in Figure 1. In Figure 2, with the ascent in the internal connection of the subgroup (from the beginning of the connection density 0.1 is 0.9512 ; the second largest eigenvalue modulus decreases slightly to 0.9561 when the number of connected nodes ascends to 100 pairs).

In Figure 3, we explore the impact of two subgroups interaction frequency on the consensus time. Frequency of interaction in the first subgroup is denoted by $p_{1}$, and the second is $p_{2}$. The interaction frequency between subgroups is denoted by $p_{3}$, which is the proportion of positive entries to all the entries in $D$ and $E$ (or the connection density between different subgroups).

In Figure 3, it is easily to be observed that if the parameter $p_{3}$ goes up, the second largest eigenvalue modulus will fall off. Thus, the consensus time will be shorter with the increasing frequency of interaction between subgroups.

3.2. The Size of Subgroups. In this section, we examine the impact of the size of subgroups on the convergence time. For a fixed size, for example, 150, of the social network, Figure 4 shows that $\left|\lambda_{2}\right|$ will be the largest when $n_{1}$ is close to $n_{2}$. More precisely, if $p_{1}=p_{2}$, when $n_{1}=n_{2}=75,\left|\lambda_{2}\right|$ will be the largest. That is to say, if a social network has a small subgroup and a large subgroup simultaneously, it will always be easy to reach a consensus. On the contrary, if the sizes of two subgroups are about the same, it is difficult to reach a consensus.

Simulation results show that the second largest eigenvalue modulus is negatively correlated to the size of the corresponding graph of $A$, as well as the density (or average degrees) of the underling graph. For convenience, they are roughly denoted by

$$
\begin{aligned}
\left|\lambda_{2}\right| & =f(n, p), \\
\frac{\partial f}{\partial n} & <0 \\
\frac{\partial f}{\partial p} & <0 .
\end{aligned}
$$




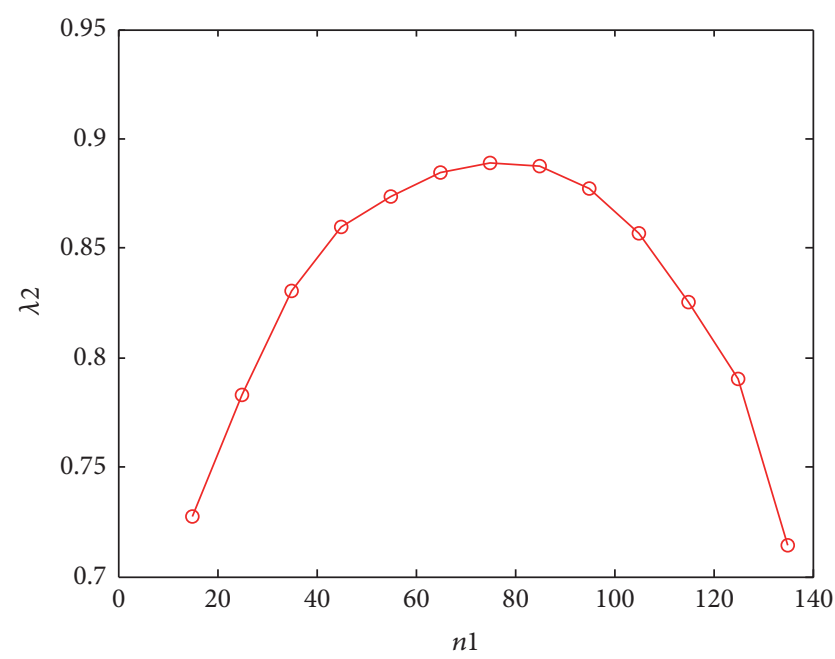

(a)

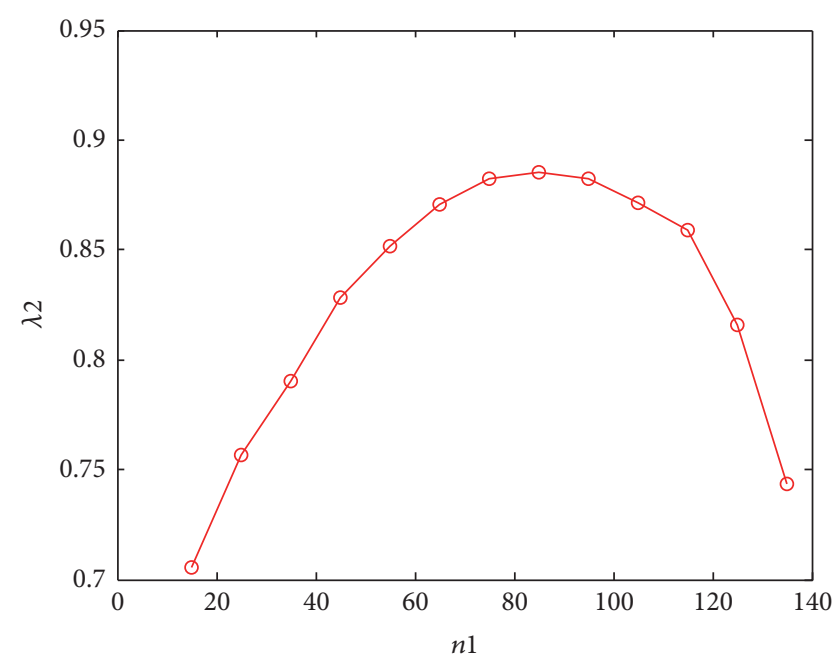

(b)

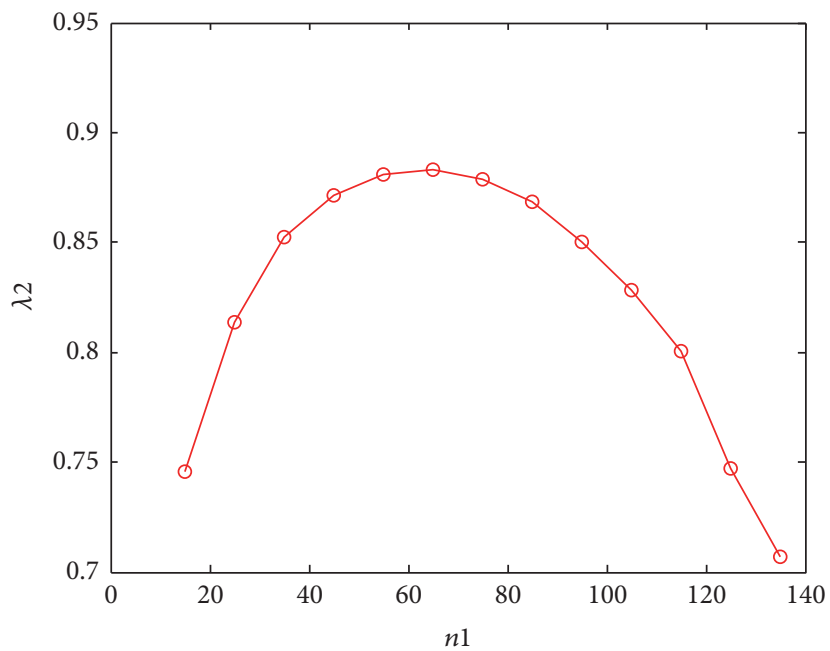

(c)

FIGURE 4: The relationship between the size of subgroups and the second largest eigenvalue modulus. $x$-axis is the size of one subgroup and $y$-axis is the second largest eigenvalue modulus. Here we assume the size of $G$ is $150, p_{3}=0.02$, in (a), $p_{1}=p_{2}=0.2 ;$ in $(\mathrm{b}), p_{1}=0.2, p_{2}=0.4$; in (c), $p_{1}=0.4, p_{2}=0.2$.

If the interaction between subgroups is very small, $A$ is nearly uncoupled; using Proposition 3 we can get

$$
\operatorname{spec}(A) \approx \operatorname{spec}(B) \cup \operatorname{spec}(C) ;
$$

thus,

$$
\begin{aligned}
\left|\lambda_{2}\right|(A) & \approx \max \left(\left|\lambda_{2}\right|(B),\left|\lambda_{2}\right|(C)\right), \\
\text { i.e., }\left|\lambda_{2}\right|(A) & \approx \max \left(f\left(n_{1}, p\right), f\left(n-n_{1}, p\right)\right) \\
& =f\left(\min \left(n_{1}, n-n_{1}\right), p\right) .
\end{aligned}
$$

So for fixed $n$, if $n_{1}=n / 2, \min \left(n_{1}, n-n_{1}\right)$ can be the largest, accordingly $\left|\lambda_{2}\right|$ is the largest.

3.3. The Distribution of Interpersonal Influence. Finally, we study three different distributions of influence strength of individuals. In Figure 5 Case 1, power law distribution represents an autocratic society, where minority-influential individuals or opinion leaders exist, and the latter two both represent a democratic society. In Case 2, the influence of individuals follows normal distribution. In Case 3, all individuals are affected by their neighbors with the same influence. Repeatedly calculating 1000 times, we can get the distribution of convergence rate as illustrated in Figure 5. It can be seen that, in an autocratic society (Case 1), the convergence of rate is slow with high probability, but sometimes is very fast. This is due to the asymmetry and heterogeneity of the power law distribution. If a few influential nodes belonging to different subgroups connect with each other, it will accelerate the convergence of groups opinions. On the contrary, if influential nodes form links only by innergroup, it will slightly slow down the convergence of group opinions. Thus, to some extent, the convergence time or convergence rate of group opinions is uncertain in this case. In a democratic society (Case 2 and Case 3 ), the convergence 


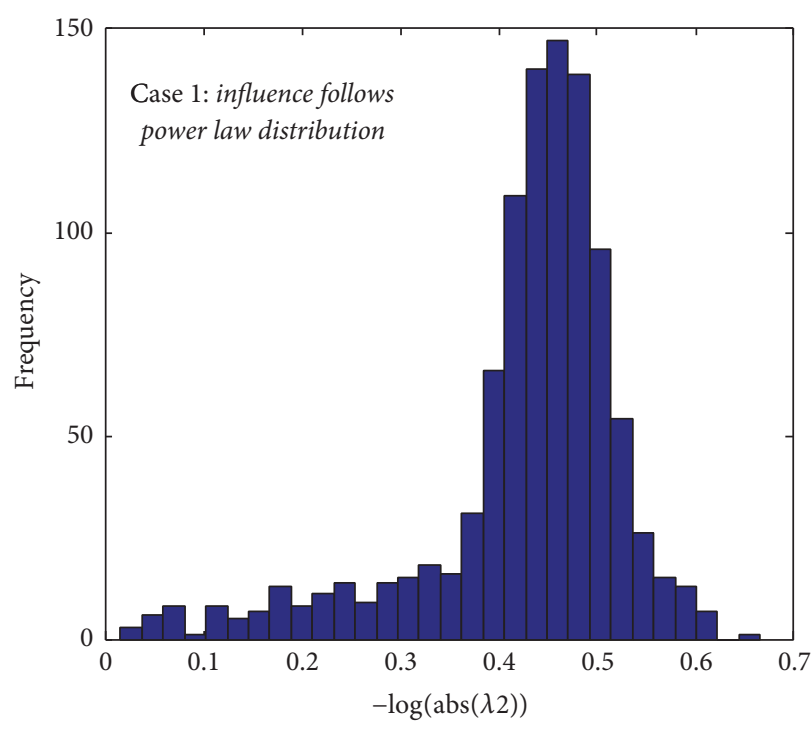

(a)

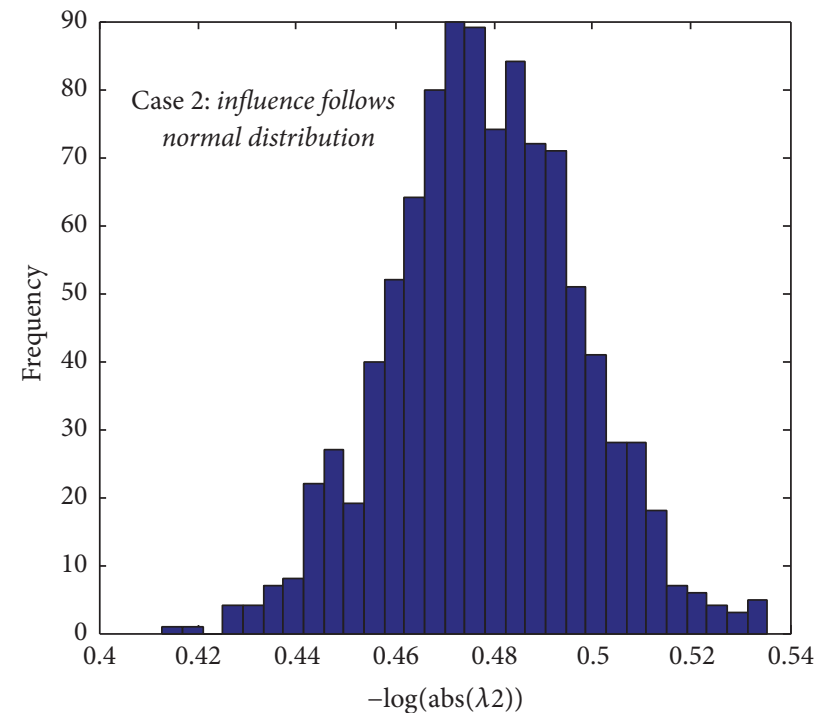

(b)

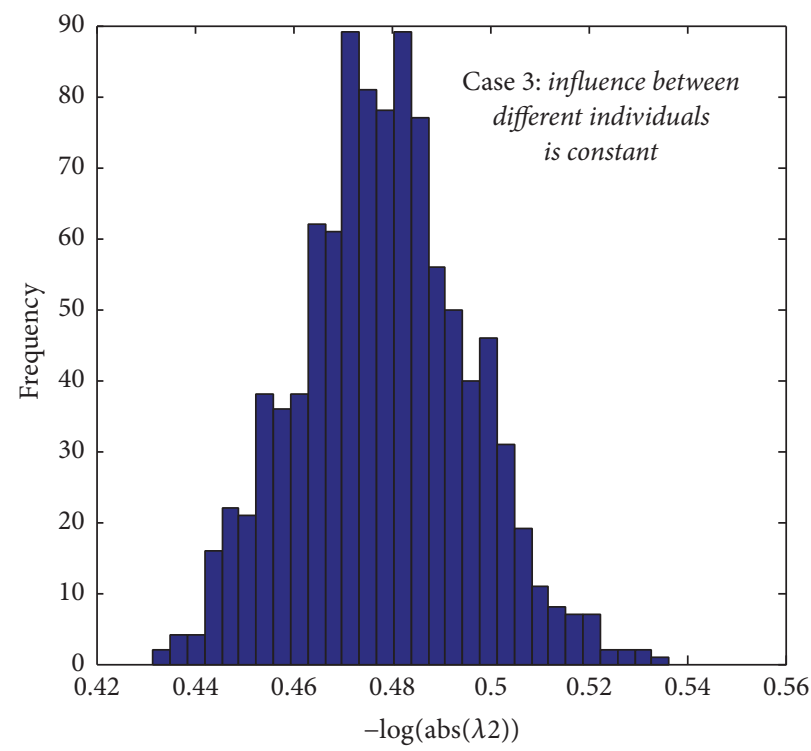

(c)

FIGURE 5: The distribution of convergence rate of group opinion is determined by the distribution of influence. $x$-axis is the convergence rate $-\log \left(\left|\lambda_{2}\right|\right)$ and $y$-axis is the frequency (total frequency is 1000). (a) represents autocratic society; (b) and (c) represent democratic society.

time follows a narrow-range distribution. In general, the average convergence rate is faster than that of Case 1. In Case 3 , the influence matrix can be expressed as $A=(D+I)^{-1} M_{A}$, where $M_{A}$ is the adjacency matrix of the graph. In this case, all the eigenvalues are real, and the second largest eigenvalue is determined by the maximum degree of the graph.

\section{Conclusions}

Based on the framework of the Degroot model, this paper studies the impact of the community structure topology on the consensus time by introducing the second largest eigenvalues modulus of social influence matrix. We prove that if the interaction between subgroups is tiny, then the consensus time will be very long. This means that opinions profiles in a social network with community structure are difficult to reach a consensus.

In order to study how the community structure impacts on the convergence time (or the convergence rate), using the second largest eigenvalue modulus, we examine this issue from three points of view: the number of nodes connected (including the number of subgroups connected, the number of subgroups within the connection, and the connection density), subgroup size, and influence distribution. We find that increasing the number of connections between subgroups can accelerate the convergence of group opinions, while increasing the number of subgroups within the subgroups slows the convergence rate. The closer the subgroups' size is, 
the longer the convergence time of the group opinions is. On the other hand, the smaller the size of the subgroup is, the shorter the convergence time is. Thus, the community structure depends not only on the density of the subgroups but also on the relative size of the subgroups. In addition, the impact of distribution of influence strength on the convergence time shows that the convergence time of group opinions in an autocratic society is longer than that in a democratic society in average, but may involve more uncertainty, which depends on the various connection pattern.

The implication of all the above is network intervene [24]. In order to accelerate the consensus, we must strengthen the communication of individuals in different subgroups. On the contrary, to maintain the discrepancy of group opinions, we can strengthen the communication of individuals in the same subgroups. Another effective way to control opinion evolution is to vary the size of subgroups.

\section{Conflicts of Interest}

The authors declare that they have no conflicts of interest.

\section{Acknowledgments}

This work was supported by State Key Development Program of Basic Research of China (2013CB329603) and the Humanity and Social Science Foundation of Ministry of Education (15YJC630111).

\section{References}

[1] S. H. Strogatz, "Exploring complex networks," Nature, vol. 410, no. 6825, pp. 268-276, 2001.

[2] S. Boccaletti, V. Latora, Y. Moreno, M. Chavez, and D.-U. Hwang, "Complex networks: structure and dynamics," Physics Reports. A Review Section of Physics Letters, vol. 424, no. 4-5, pp. 175-308, 2006.

[3] R. Pastor-Satorras and A. Vespignani, "Epidemic spreading in scale-free networks," Physical Review Letters, vol. 86, no. 14, pp. 3200-3203, 2001.

[4] R. Pastor-Satorras, C. Castellano, P. Van Mieghem, and A. Vespignani, "Epidemic processes in complex networks," Reviews of Modern Physics, vol. 87, no. 3, pp. 925-979, 2015.

[5] M. Nekovee, Y. Moreno, G. Bianconi, and M. Marsili, "Theory of rumour spreading in complex social networks," Physica A: Statistical Mechanics and Its Applications, vol. 374, no. 1, pp. 457470, 2007.

[6] F. Amblard and G. Deffuant, "The role of network topology on extremism propagation with the relative agreement opinion dynamics," Physica A: Statistical Mechanics and its Applications, vol. 343, no. 1-4, pp. 725-738, 2004.

[7] S. Boccalett et al., "The structure and dynamics of multilayer networks," Physics Reports, vol. 544, no. 1, pp. 1-122, 2014.

[8] M. Meadows and D. Cliff, The Relative Agreement Model of Opinion Dynamics in Populations with Complex Social Network Structure: The Fourth International Workshop on Complex Networks, Berlin, Germany, 2013.

[9] K. Sznajd-Weron and J. Sznajd, "Opinion evolution in closed community," International Journal of Modern Physics C, vol. 11, no. 6, pp. 1157-1165, 2000.
[10] P. Clifford and A. Sudbury, "A model for spatial conflict," Biometrika, vol. 60, pp. 581-588, 1973.

[11] R. Holley and T. M. Ligget, "Ergodic thermos for weakly interacting system and the voter model," The Annals of Probability, vol. 3, no. 4, pp. 643-663, 1975.

[12] S. Galam, "Majority rule, hierarchical structures, and democratic totalitarianism: A statistical approach," Journal of Mathematical Psychology, vol. 30, no. 4, pp. 426-434, 1986.

[13] B. Latané, "The psychology of social impact," American Psychologist, vol. 36, no. 4, pp. 343-356, 1981.

[14] G. Deffuant, D. Neau, F. Amblard, and G. Weisbuch, "Mixing beliefs among interacting agents," Advances in Complex Systems, vol. 3, no. 04, pp. 87-98, 2000.

[15] R. Hegselmann and U. Krause, "Opinion dynamics and bounded confidence models, analysis, and simulations," Journal of Artificial Societies and Social Simulation, vol. 5, no. 3, 2002, http://jasss.soc.surrey.ac.uk/9/1/8.html.

[16] M. H. DeGroot, "Reaching a consensus," Journal of the American Statistical Association, vol. 69, no. 345, pp. 118-121, 1974.

[17] R. Olfati-Saber and R. M. Murray, "Consensus problems in networks of agents with switching topology and time-delays," Institute of Electrical and Electronics Engineers. Transactions on Automatic Control, vol. 49, no. 9, pp. 1520-1533, 2004.

[18] P.-P. Li, D.-F. Zheng, and P. M. Hui, "Dynamics of opinion formation in a small-world network," Physical Review E-Statistical, Nonlinear, and Soft Matter Physics, vol. 73, no. 5, Article ID 056128, 2006.

[19] P. L. Krapivsky and S. Redner, "Dynamics of majority rule in two-state interacting spins systems," Physica Review Letter, vol. 90, no. 23, Article ID 238701, 2003.

[20] V. Sood and S. Redner, "Voter model on heterogeneous graphs," Physical Review Letters, vol. 94, no. 17, Article ID 178701, 2005.

[21] P. Singh, S. Sreenivasan, B. K. Szymanski, and G. Korniss, "Accelerating consensus on coevolving networks: The effect of committed individuals," Physical Review E-Statistical, Nonlinear, and Soft Matter Physics, vol. 85, no. 4, Article ID 046104, 2012.

[22] D. J. Hartfiel and C. D. Meyer, "On the structure of stochastic matrices with a subordinant eigenvalue near 1," Linear Algebra and its Applications, vol. 272, pp. 193-203, 1998.

[23] D. J. Watts and S. H. Strogatz, "Collective dynamics of 'smallworld' networks," Nature, vol. 393, no. 6684, pp. 440-442, 1998.

[24] T. W. Valente, "Network interventions," Science, vol. 336, no. 6090, pp. 49-53, 2012. 


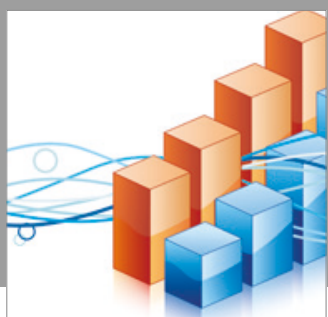

Advances in

Operations Research

vatersals

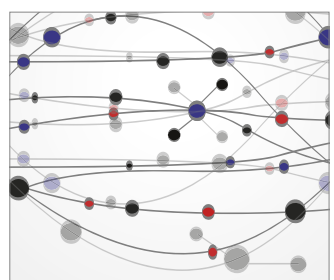

\section{The Scientific} World Journal
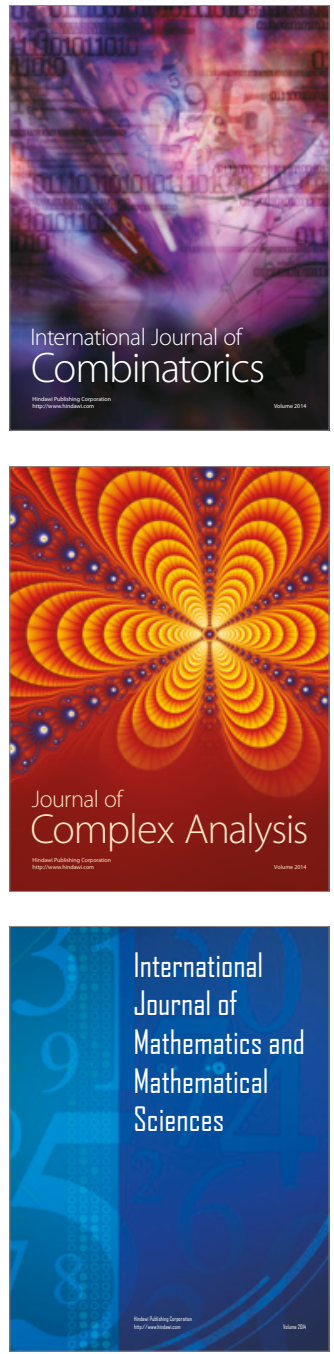
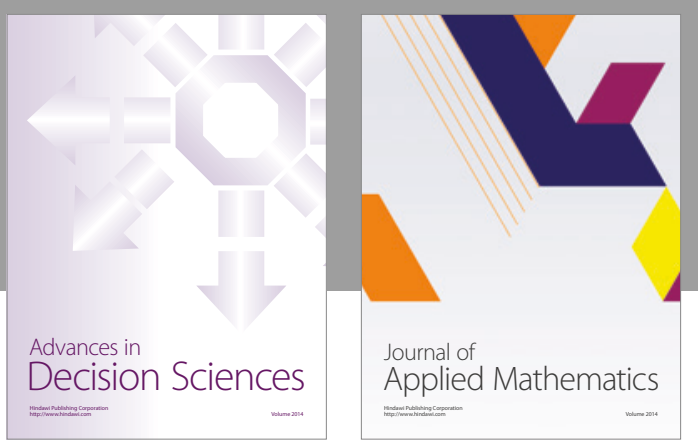

Algebra

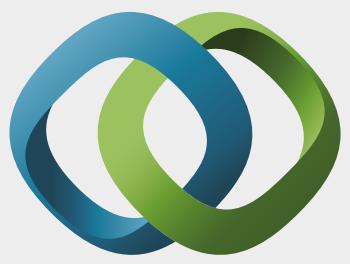

\section{Hindawi}

Submit your manuscripts at

https://www.hindawi.com
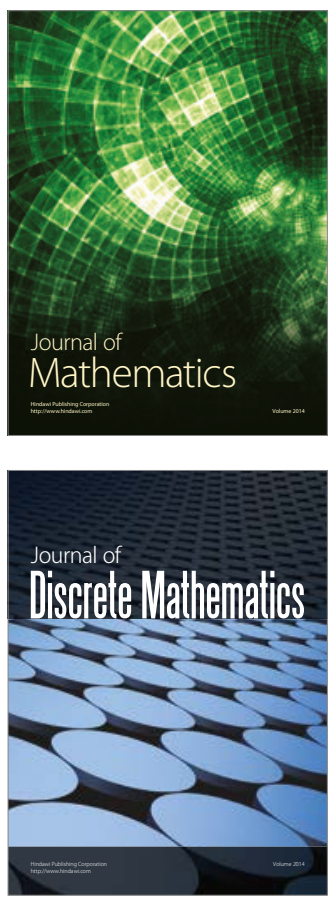

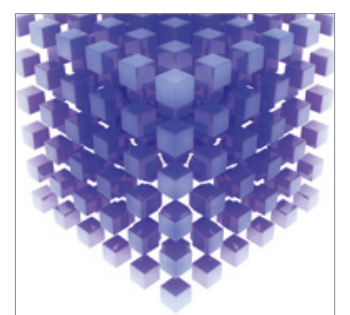

Mathematical Problems in Engineering
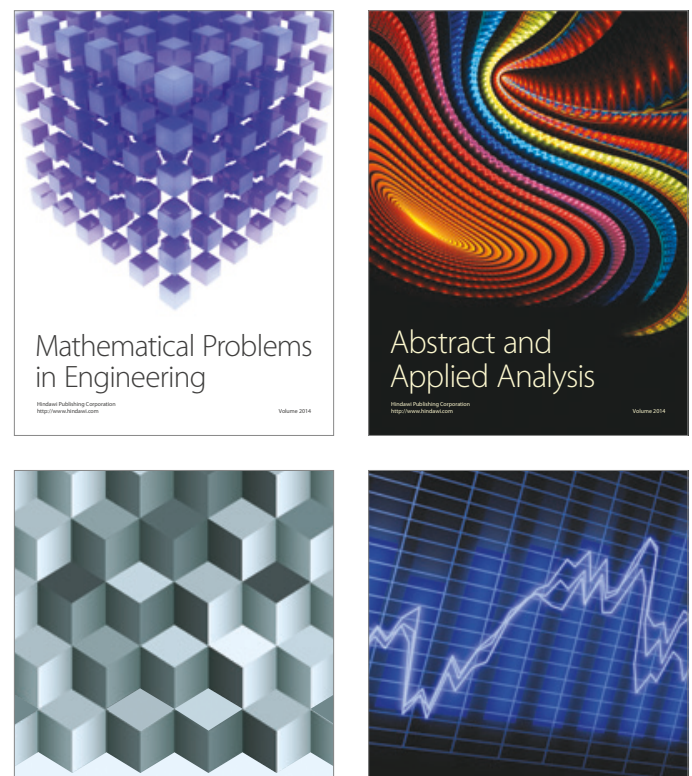

Journal of

Function Spaces

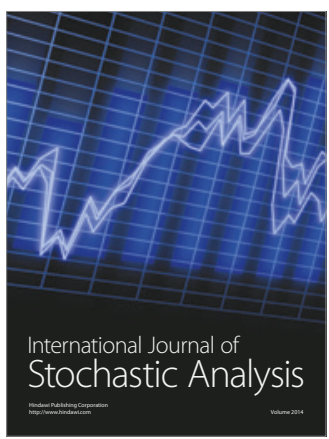

Probability and Statistics
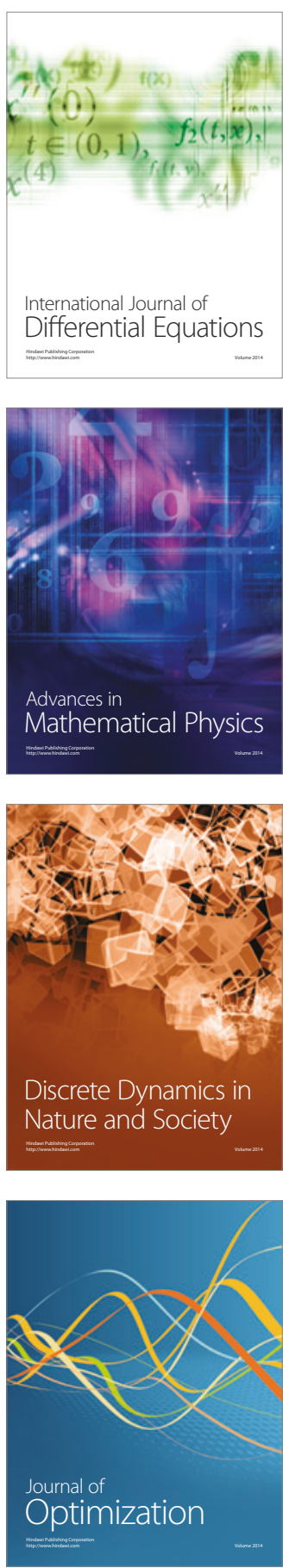